\title{
Dynamic Traffic Feedback Data Enabled Energy Management in Plug-in Hybrid Electric Vehicles
}

\author{
Chao Sun, Scott J. Moura, Member, IEEE, Xiaosong Hu, Member, IEEE, J. Karl Hedrick, and Fengchun Sun
}

\begin{abstract}
Recent advances in traffic monitoring systems have made real-time traffic velocity data ubiquitously accessible for drivers. This paper develops a traffic data-enabled predictive energy management framework for a power-split plug-in hybrid electric vehicle (PHEV). Compared with conventional model predictive control (MPC), an additional supervisory state of charge (SOC) planning level is constructed based on real-time traffic data. A power balance-based PHEV model is developed for this upper level to rapidly generate battery SOC trajectories that utilized as final state constraints in the MPC level. This PHEV energy management framework is evaluated under three different scenarios: (i) without traffic flow information, (ii) with static traffic flow information, and (iii) with dynamic traffic flow information. Numerical results using real-world traffic data illustrate that the proposed strategy successfully incorporates dynamic traffic flow data into the PHEV energy management algorithm to achieve enhanced fuel economy.
\end{abstract}

Index Terms-Traffic Velocity, Fuel Economy, Plug-in Hybrid Electric Vehicle, Supervised Energy Management, Power Balance Model.

\section{INTRODUCTION}

\section{A. Motivation}

$\mathbf{T}$ RAFFIC monitoring systems are now ubiquitous components of modern intelligent transportation systems [1], [2]. Many types of sensors, such as loop detectors, pneumatic sensors, and cameras, are employed to monitor traffic conditions [3]. Several traffic monitoring projects, such as the Caltrans Performance Measurement System (PeMS) [4] and Houston TranStar [5], have been successfully implemented and currently provide real-time traffic data on the Internet. As such, access to real-time traffic data is now fairly ubiquitous. This information complements existing in-vehicle navigation systems that include GPS [6] and 3-D terrain maps [7], [8]. These information systems provide the opportunity to incorporate more environmental information than ever before into PHEV energy management [9], [10]. This paper systematically integrates real-time traffic velocity data into the energy management of a power-split PHEV.

In the energy management problem (EMP) of PHEVs, SOC is an important state in determining the optimal power split

C. Sun is a Ph.D. student of National Engineering Laboratory for Electric Vehicles, Beijing Institute of Technology, Beijing 100081, China. Now he is a visiting student researcher of Department of Mechanical Engineering, University of California, Berkeley, CA 94720, USA (chaosun.email@gmail.com).

S. Moura and X. Hu are with the Department of Civil and Environmental Engineering, University of California, Berkeley, CA 94720, USA (smoura@berkeley.edu,xiaosong@chalmers.se).

J. K. Hedrick is with the Department of Mechanical Engineering, University of California, Berkeley, CA 94720, USA (khedrick@me.berkeley.edu).

F. Sun is with the National Engineering Laboratory for Electric Vehicles, Beijing Institute of Technology, Beijing 100081, China (sunfch@bit.edu.cn).

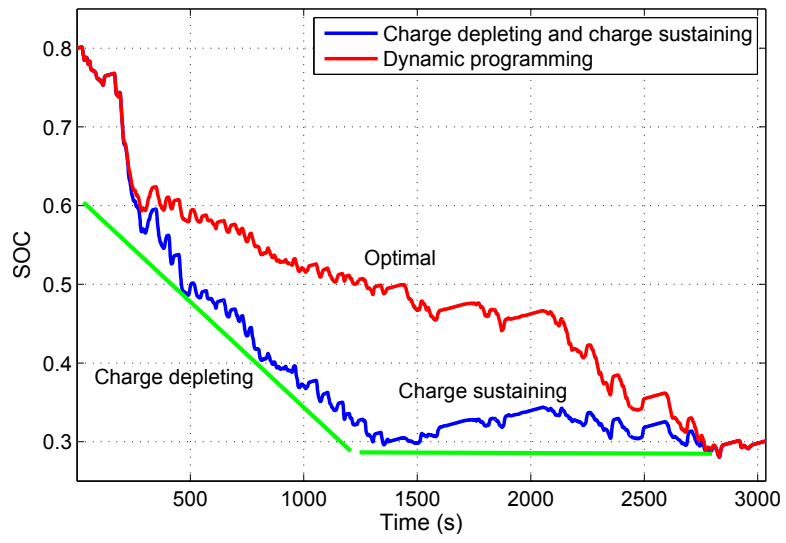

Fig. 1. SOC trajectories derived from the CDCS strategy and DP.

ratio between the engine and battery [11]. If no future driving information is available, the charge depleting and charge sustaining (CDCS) strategy is commonly used [12], [13], as shown by the blue curve in Fig. 1. When the trip distance exceeds the all-electric range, CDCS is suboptimal [14]. As can be seen in Fig. 1, the optimal SOC trajectory solved by dynamic programming (DP) differs significantly from CDCS [15]. In particular, the CDCS strategy consumes $22.17 \%$ more fuel than DP. Given more information about the velocity profile - enabled from traffic data - we hypothesize that a near-optimal energy management strategy can be developed.

Previous work has examined the EMP for pre-planned driving tasks. The authors of [16] developed a SOC reference generator for hilly driving profiles, and demonstrated improved fuel economy. In this paper, we consider a SOC pre-planning approach under time-varying traffic conditions. Consider the traffic map in Fig. 2, which displays the instantaneous traffic flow velocity in the San Francisco Bay Area according to Google Maps at 9:15 AM, on Feb 3, 2014. Red road segments indicate slow traffic velocity from a congestion event. If a PHEV knows when and where the congestion occurs, then the controller can pre-plan a corresponding optimal SOC trajectory and adjust the energy management strategy accordingly. As shown by the block diagram in Fig. 2, real-time traffic data is utilized for SOC planning and control. Meanwhile, vehicle velocities are continuously monitored and fed back into the traffic monitoring system. Consequently, this paper adds dynamic traffic feedback to the existing literature on PHEV energy management [15], [17], including DP [18], equivalent consumption minimization strategy (ECMS) [19] and model predictive control (MPC) [20]. 


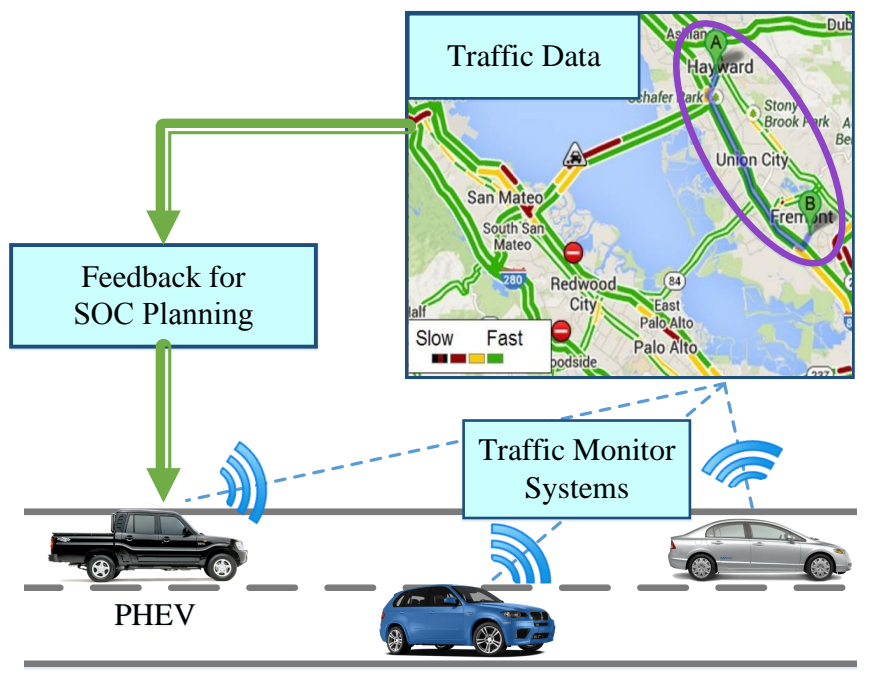

Fig. 2. Traffic data feedback framework, and the traffic flow velocity provided by Google Maps on 02/03/2014, 9:15 AM. Road colors indicate the traffic flow velocities. The area marked by the purple ellipse is the experiment field for traffic data collecting (see Section II).

\section{B. Literature Review of PHEV Energy Management}

Most studies that employ DP for energy management assume that the entire driving profile and terrain information is fully known [21], [22]. DP provides a provably optimal energy management strategy. However, exact knowledge of the driving profile is seldom known in practice. As a result, DP solutions are mainly used as benchmarks for optimality [23]. In many cases, statistics about routine driving profiles, e.g. bus routes or everyday commutes, are known beforehand and can be reliably modeled via Markov chains [24], [25]. Iterative learning also shows excellent performance in predicting driving profiles for repeatable trips [26]. Nevertheless, these methods perform poorly when the driving route changes dramatically, e.g. due to traffic congestion, or no historical data is available.

ECMS is an established approach that determines the optimal power split at each time instant rather than over a time horizon [19], [27]. Therefore, no future driving information is needed. Given an appropriate equivalence factor, ECMS can achieve near-optimal fuel economy [14], [28]. However, tuning the equivalence factor is nontrivial and there is no guarantee that ECMS produces globally optimal performance. If the driving profile can be recognized in real time, ECMS can adjust the equivalence factor accordingly to achieve better fuel economy via Adaptive-ECMS [27], [29].

MPC can be viewed as a compromise between DP and ECMS. That is, MPC minimizes a series of cost functions over receding time horizons [30], whereas DP and ECMS optimize over global and instantaneous cost functions, respectively. The predictive ability of MPC over instantaneous approaches helps achieve better performance. This attractive property has generated increased attention from automobile industry practitioners [31], [32]. In the literature, many researchers have examined MPC to solve the EMP for HEV/PHEVs. Nonlinear programming [33], quadratic programming [34], Pontryagin's minimum principle [35], and DP [20], [36] have all been applied. These predictive energy management strategies provide improved performance over instantaneous or rule-based energy management strategies, and are real-time implementable [17]. However, to the authors' best knowledge, the study of dynamic traffic feedback data enabled MPC energy management for PHEVs has not been investigated.

\section{Main Contributions}

The main contribution of this paper is a traffic dataenabled predictive control framework for PHEV energy management, to achieve near optimal fuel consumption. Compared with conventional MPC, this framework includes a higher supervisory battery SOC planning level, aiming to improve the controller performance from a global perspective. A power balance PHEV model is developed for reference SOC trajectory generation based on the obtained traffic data. Compared with conventional PHEV models, the power balance model significantly reduces SOC trajectory computation time, thereby enabling real-time implementation at updates rates commensurate with traffic data. In this paper, a realworld highway driving scenario is used for validation and is based on collected traffic flow data from the Mobile Century project [37]. Although the foregoing contributions are made specifically for a power-split PHEV in a highway driving scenario, the proposed approach extends to other HEV/PHEV configurations or other driving situations when traffic velocity information is available.

\section{Outline}

The remainder of the paper is arranged as follows. In Section II, the obtained traffic data is presented and analyzed. Section III introduces the supervised predictive energy management strategy. Section IV details the control-oriented PHEV model and formulates the nonlinear control problem. Simulation results are illustrated in Section V, followed by key conclusions in Section VI.

\section{Traffic Flow Velocity Information}

Large scale traffic monitoring systems have been deployed across the world. Reference [37] presents a traffic monitoring system, nicknamed Mobile Century, based on GPS-enabled smartphones. This system exploits the extensive coverage of the cellular network, position and velocity measurements of GPS, and the communication ability of cellphones. A field experiment was conducted to measure traffic velocities and flow on a 10-mile stretch of I-880 near Union City, California, for 8 hours, as shown in Fig. 2 between points A and B. We leverage this data to study traffic-enabled PHEV energy management in this paper.

\section{A. Traffic Flow Velocity Extraction}

The Mobile Century vehicles were all equipped with GPSenabled smartphones that produce time-stamped position and velocity measurements every 3 seconds. A traffic estimation server is used to collect all the GPS data and analyze traffic 


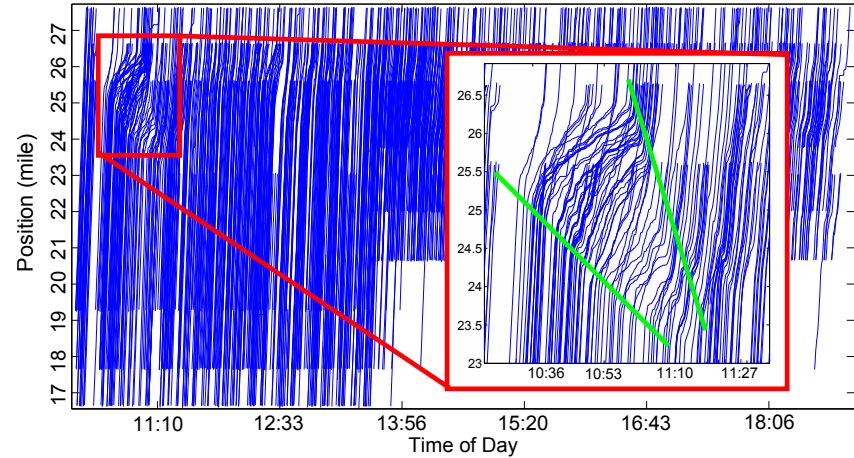

Fig. 3. Time-position trajectories of individual trips from the Mobile Century field experiment on highway road I-880, from 16 to 28 mile north bound.

flow dynamics. Figure 3 plots the time-position trajectory for each trip in the field experiment. A traffic congestion event can be identified within the red rectangle marked in the figure. All vehicles were forced to decelerate when driving through the congestion area. Over time, the congestion attenuates and vehicles gradually accelerated back to normal speeds. The propagation of a shock-wave can be observed clearly.

The traffic congestion period, from 10:20 AM to 13:40 PM is selected deliberately as the object of study. This is because a traffic congestion event can fully reflect the uncertainty and complexity of the driving environment. Note that the flow velocity data received by the drivers is only a static reflection of the traffic. The information is more valuable when it updates in real-time. In this paper, we assume the driver can obtain the traffic velocity data every 300 seconds, which is consistent with the update rate of PeMS [4]. The road is split into 120 segments for vehicle velocity sampling (160 meters per segment which is approximately the length of a postmile interval in California). The average velocity of all vehicles that drive through a specific road segment is assumed to be the traffic flow velocity of this segment, which is calculated by

$$
V_{\text {avg }}^{S I}(t)=\frac{\sum_{i=1}^{N} V_{i}^{S I}(t)}{N}, \quad(S I=1,2, \ldots, 120) .
$$

where $V_{a v g}^{S I}(t)$ indicates the average velocity of the $S I$ th (segment index) road segment at time $t, V_{i}^{S I}(t)$ is the velocity of one individual vehicle and $N$ represents the total number of vehicles driving through segment $S I$.

\section{B. Trip Analysis and Processing}

The extracted traffic velocity is depicted in Fig. 4. Note that velocity reduces from $70 \mathrm{mph}$ to less than $10 \mathrm{mph}$ near the 24-mile position at 10:45 AM, which is the beginning of the traffic congestion event. The velocity of the congested segment starts to increase around 11:20 AM, which is the end of the traffic congestion event.

We assume the PHEV follows the same velocity profile as the traffic flow velocity. Due to low spatial resolution, the extracted traffic velocity profile is piecewise constant. We smooth $V_{a v g}$ with a Butterworth filter to produce a more

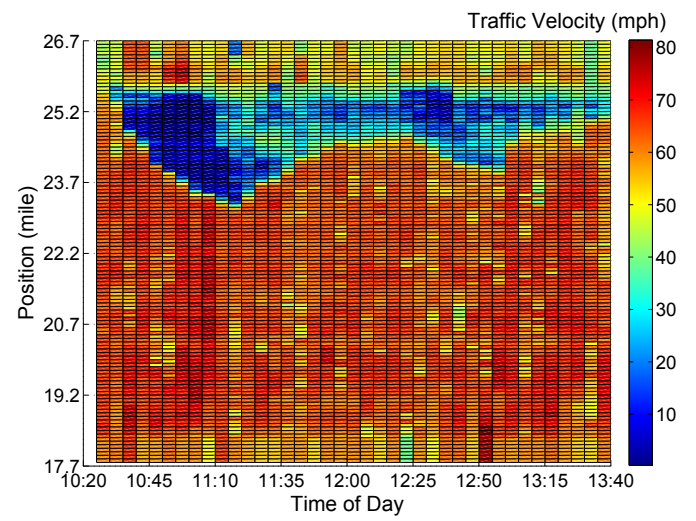

Fig. 4. Extracted traffic flow velocity distribution from 10:20 AM to 13:40 PM. Sampling time interval is 300 seconds and the road resolution is 0.1 mile.

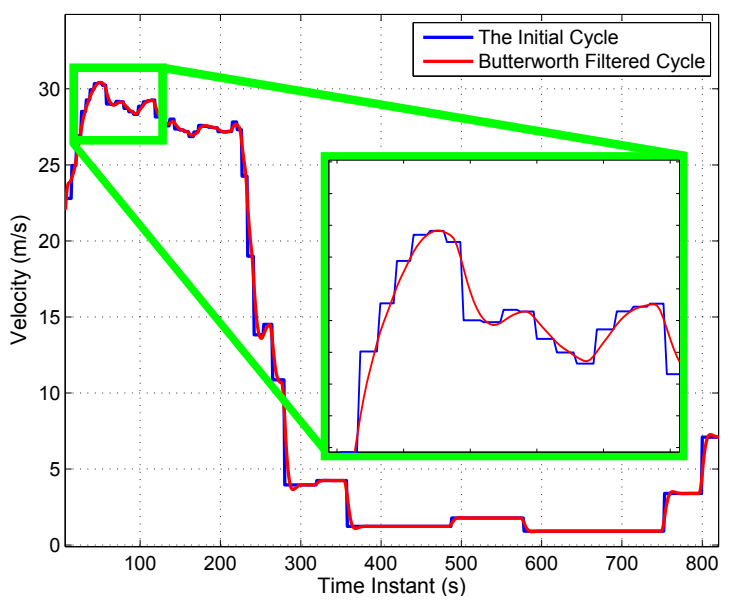

Fig. 5. Extracted velocity profile example, before and after filtering.

realistic and trackable velocity signal ( $V_{\text {flow }}$ in Section III Part A). Fig. 5 illustrates an extracted and filtered velocity profile. We can see that the extracted profile is piecewise constant (blue line). The red curve is the filtered result, which is smooth and follows the original cycle reasonably well. Each time the traffic flow velocity information is received, the velocity profile is filtered.

\section{Supervised Predictive Energy Management}

\section{A. Traffic Enabled Energy Management}

The structure of the supervised predictive energy management is demonstrated in Fig. 6. In the upper level, the traffic flow velocity $V_{\text {flow }}$ obtained from real-time traffic data is utilized to calculate the optimal SOC trajectory. In the lower level, a horizon velocity predictor is employed to forecast the future driving velocities in each receding horizon. These two aspects correspond to the long-term and short-term disturbances, respectively.

It is important to note that the acquired traffic flow velocity will differ from the actual vehicle velocity. Thus, errors exist in the generated SOC reference trajectory. The generated SOC trajectory is introduced into the MPC level as a terminal SOC 


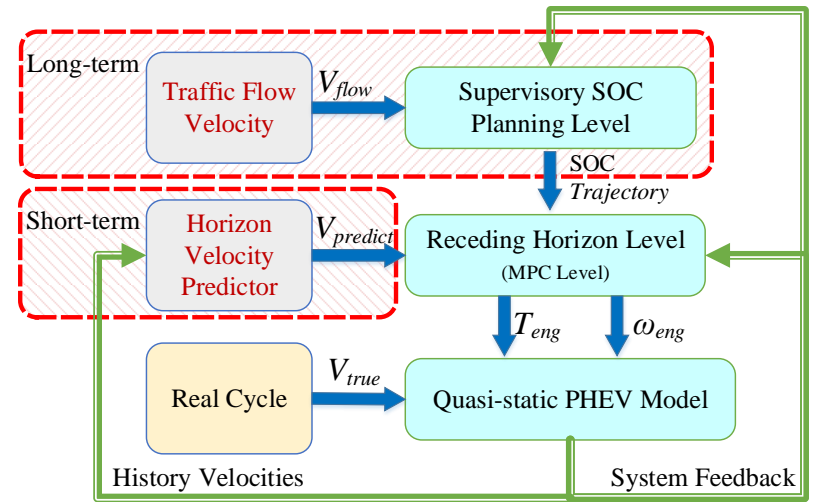

Fig. 6. Control structure for the supervised predictive energy management scheme. The upper level uses real-time traffic flow velocity to compute a global SOC trajectory. The lower level applies a receding horizon control, using the SOC trajectory as a final state constraint and a short-term velocity predictor.

reference during each control horizon. This provides the lowerlevel MPC feedback loop with additional control flexibility to compensate for SOC reference errors, denoted as

$$
\overline{\mathrm{SOC}}=\mathrm{SOC}^{*}
$$

where $\overline{\mathrm{SOC}}$ is the terminal SOC reference of each control horizon and $\mathrm{SOC}^{*}$ is the generated optimal SOC trajectory. The detailed control procedure is described as below:

- Acquire real-time traffic data and route information, calculate the optimal battery SOC trajectory;

- Predict the short-term future velocity profile for the current control horizon;

- Given the SOC reference and the predicted velocity sequence $V_{\text {predict }}$, the MPC controller calculates the optimal control policy;

- Apply the first time-step of the optimal control policy in the quasi-static PHEV model;

- Feed back system states, update system constraints, and repeat the computation procedure at the next time instant.

Note that step 1 of the control procedure is activated only when the traffic and route information is updated. Also note that the upper and lower levels utilize different PHEV models for optimization. In the upper level, a simple power-based model is utilized for SOC trajectory generation. In the lower level, a higher fidelity PHEV model is used for MPC. We describe each level in the following subsections. The quasistatic PHEV model is a detailed plant model furnished by the QSS-toolbox developed at ETH Zürich, which has been validated against experiments (see [38] for details).

\section{B. Long-term SOC Trajectory Generation}

The optimal SOC trajectory calculation must be fast enough to follow the traffic flow dynamics. The commonly used control-oriented PHEV model [18], [21] is proficient for powertrain control, but excessively complex for SOC trajectory calculation for this particular purpose. This paper introduces a reduced power balance based PHEV model for this purpose.

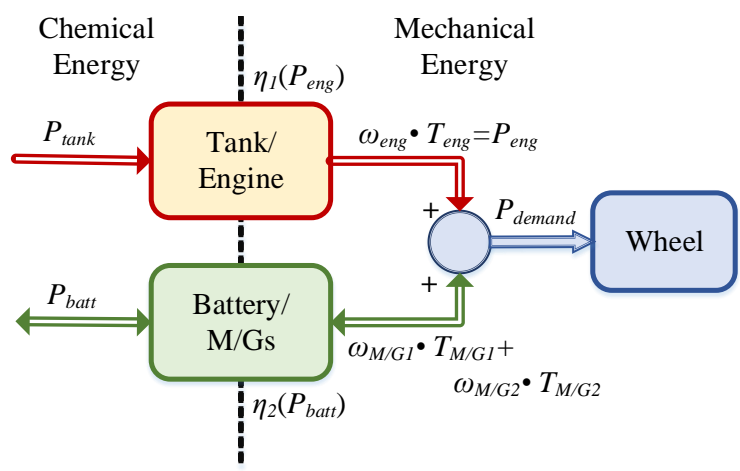

Fig. 7. Structure of the simplified power-split PHEV powertrain.

This model is much more computationally efficient, yet sufficiently accurate for the purpose of SOC trajectory calucations.

The power balance model is based on a simplified powersplit PHEV powertrain, as shown in Fig. 7. Instead of establishing physical models for all the powertrain components, the power balance model only describes power flows and conversion efficiencies. The tank/engine converts chemical energy into mechanical energy with efficiency denoted by $\eta_{1}$. The battery and two motor/generators (M/Gs) convert between chemical battery energy and mechanical energy with efficiency denoted by $\eta_{2}$. The sum of the engine and M/Gs' mechanical powers must equal power demand, $P_{\text {demand }}$.

Mathematically, the simplified powertrain is governed by power balance equation

$$
\eta_{1} P_{\text {tank }}(t)+\eta_{2} P_{\text {batt }}(t)=P_{\text {demand }}(t)
$$

where $P_{\text {tank }}$ is the chemical energy supplied by the tank, $P_{b a t t}$ is the electro-chemical energy supplied by the battery and $P_{\text {demand }}$ is the vehicle power demand. Positive $P_{b a t t}$ denotes discharging. Parameters $\eta_{1}$ and $\eta_{2}$ are synthetic energy conversion coefficients of the mechanical propulsion path (engine side) and the electrical propulsion path (M/G side), respectively. The power demand is provided by

$$
P_{\text {demand }}(t)=\left(m a+C_{r} m g+\frac{1}{2} \rho A C_{d} v^{2}(t)\right) v(t)
$$

where $m$ is the vehicle mass, $a$ is the vehicle acceleration, $g$ is gravitational acceleration, $v(t)$ is the vehicle velocity, $C_{r}$ represents the rolling resistance coefficient and $\frac{1}{2} \rho A C_{d}$ is the aerodynamic drag resistance. The battery package is modeled as an equivalent circuit [39]. The battery power and the battery SOC is derived with

$$
\begin{aligned}
& P_{\text {batt }}(t)=V I_{\text {batt }}(t)-I_{\text {batt }}^{2}(t) R \\
& \operatorname{SOCC}(t)=-\frac{I_{\text {batt }}(t)}{Q}
\end{aligned}
$$

where $V$ and $R$ are the open circuit voltage and internal resistance, respectively; $I_{b a t t}(t)$ and $Q$ are the battery current and capacity, respectively.

In this power balance PHEV model, the number of control variables is reduced from two to one compared with the control-oriented model (to be presented in Section IV). 
Battery output power is selected as the control variable, where $u=P_{b a t t}$. Battery SOC and the engine on/off state (denoted as $\mathrm{O}$ ) are selected as state variables, where $x=[\mathrm{SOC}, \mathrm{O}]^{T}$. $\mathrm{O}=1$ means the engine is on, and $\mathrm{O}=0$ means the engine is off. Define switching of the engine state as

$$
\delta \mathrm{O}(k \Delta t)=|\mathrm{O}(k \Delta t)-\mathrm{O}((k-1) \Delta t)|
$$

The cost function is formulated as

$$
J=\int_{0}^{T}\left[P_{\text {tank }}(u(t))+w \delta \mathbf{O}(t)\right]^{2} d t
$$

where $P_{\text {tank }}(u(t))$ penalizes fuel consumption and $w$ is the penalty for engine state switching. The expression for $P_{\text {tank }}(u(t))$ is derived as follows. From (3) we have

$$
P_{\text {tank }}(t)=\frac{P_{\text {demand }}(t)-\eta_{2} P_{\text {batt }}(t)}{\eta_{1}} .
$$

Note that $u(t)=P_{\text {batt }}(t)$. Moreover, consider the conversion efficiencies with the following arguments

$$
\begin{aligned}
& \eta_{1}=\eta_{1}\left(P_{\text {eng }}\right), \\
& \eta_{2}=\eta_{2}\left(P_{\text {batt }}\right)=\eta_{2}(u) .
\end{aligned}
$$

Note that $P_{\text {eng }}=P_{\text {demand }}-\eta_{2} P_{\text {batt }}=P_{\text {demand }}-\eta_{2}(u) \cdot u$. Consequently, we obtain

$$
P_{\text {tank }}(t)=\frac{P_{\text {demand }}(t)-\eta_{2}(u(t)) \cdot u(t)}{\eta_{1}\left(P_{\text {eng }}(t)\right)}=\frac{P_{\text {eng }}(t)}{\eta_{1}\left(P_{\text {eng }}(t)\right)}
$$

DP is used to minimize $J$, subject to

$$
\begin{aligned}
& \mathrm{SOC}^{\mathrm{min}} \leq \mathrm{SOC} \leq \mathrm{SOC}^{\max }, P_{\text {tank }}^{\min } \leq P_{\text {tank }} \leq P_{\text {tank }}^{\max } \\
& I_{\text {batt }}^{\min } \leq I_{\text {batt }} \leq I_{\text {batt }}^{\max }, P_{\text {batt }}^{\min } \leq P_{\text {batt }} \leq P_{\text {batt }}^{\max } .
\end{aligned}
$$

The energy conversion coefficients $\eta_{1}$ and $\eta_{2}$ are very important in describing the nonlinearity of the powertrain. Efficiency $\eta_{1}$ can be calculated by assuming the engine always operates on the optimum operating line (OOL). In contrast, $\eta_{2}$ involves the combined operating efficiencies of both M/G1 and $\mathrm{M} / \mathrm{G} 2$, and it is therefore not possible to assume operation along the individual OOLs. Thus, this paper proposes an empirical approach to determine $\eta_{1}$ and $\eta_{2}$, based on the optimal operating behaviors yielded from the control-oriented model. Details are shown below:

- First, utilize DP to solve the EMP with the controloriented PHEV model across a variety of driving cycles;

- Second, collect the optimal solutions for powertrain behavior analysis. Coefficients $\eta_{1}$ and $\eta_{2}$ are calculated by

$$
\begin{aligned}
\eta_{1} & =\frac{\omega_{\text {eng }} \cdot T_{\text {eng }}}{P_{\text {tank }}} \\
\eta_{2} & =\frac{\omega_{M / G 1} \cdot T_{M / G 1}+\omega_{M / G 2} \cdot T_{M / G 2}}{P_{\text {batt }}}
\end{aligned}
$$

where $\omega_{e n g}, \omega_{M / G 1}, \omega_{M / G 2}$ and $T_{e n g}, T_{M / G 1}, T_{M / G 2}$ are corresponding rotation speeds and torques of the engine, M/G1 and M/G2 from the control-oriented model.

- Last but most important, formulate $\eta_{1}$ and $\eta_{2}$ by least squares curve fitting.

In step one, four driving cycles, including both urban and highway types, are used for the optimal energy management simulation: WVUCITY, NYCC, Artemis-highway

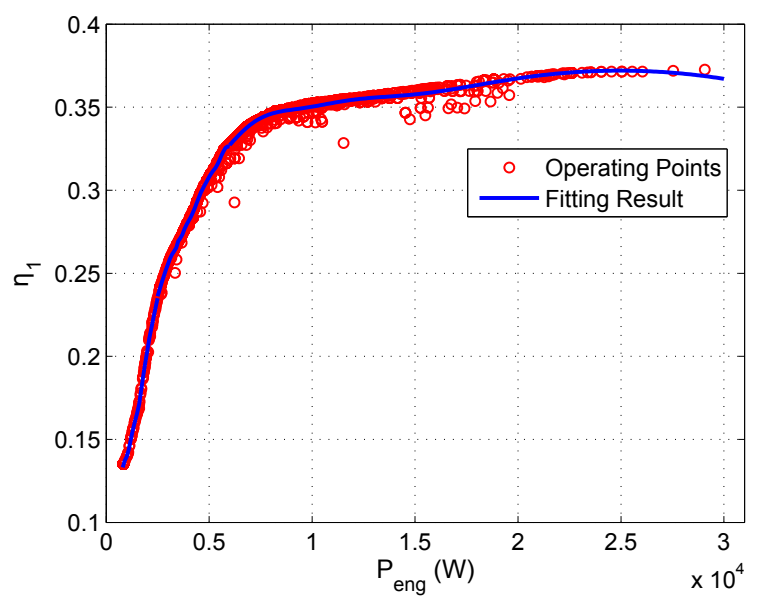

Fig. 8. The $P_{\text {eng }}-\eta_{1}$ operating points and the curve fitting result, $\eta_{1}=0$ when $0 \leq P_{\text {eng }}<\min$.

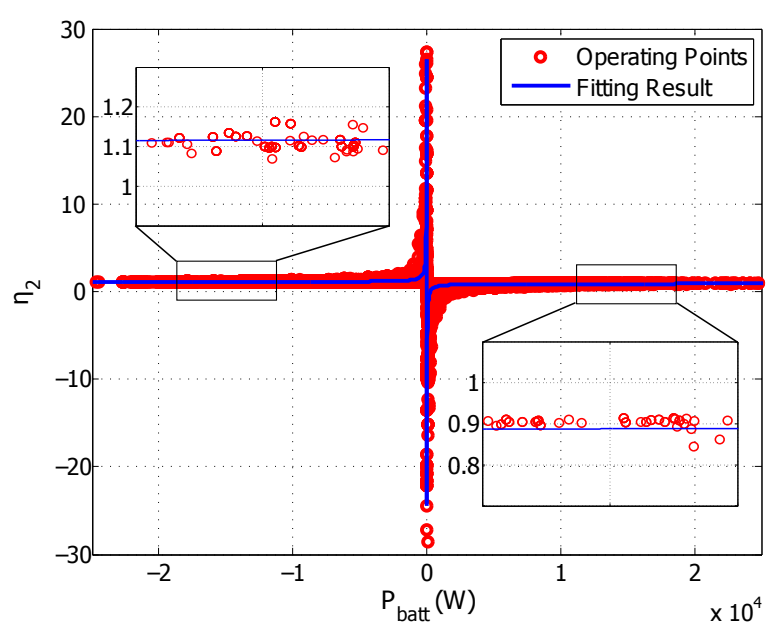

Fig. 9. The $P_{b a t t}-\eta_{2}$ operating points and the curve fitting result.

and HWFET provided by the U.S. Environmental Protection Agency and [40]. In order to cover as much of the operating envelope as possible, different battery discharging depths are investigated.

The operating points for functions $\eta_{1}$ and $\eta_{2}$ are plotted in Fig. 8 and Fig. 9. It can be seen that $\eta_{1}$ and $\eta_{2}$ are strongly correlated with $P_{e n g}$ and $P_{b a t t}$, respectively. Consequently, the hypotheses in (10) and (11) are verified. Interestingly, the fitting results for $\eta_{1}$ prove to be consistent with the engine OOL approach. Piecewise functions are employed for curve fitting of $\eta_{2}$, including polynomial functions and mixture Gaussian functions.

The complete power balance-based model is validated in Section V-A against a more detailed control-oriented model. Next we consider the issue of velocity prediction in the MPC level from Fig 6.

\section{Short-term Velocity Prediction}

Next we develop a forecasting technique to predict shortterm vehicle velocities. This paper employs a data driven approach to velocity prediction. Vehicles with forward radar 


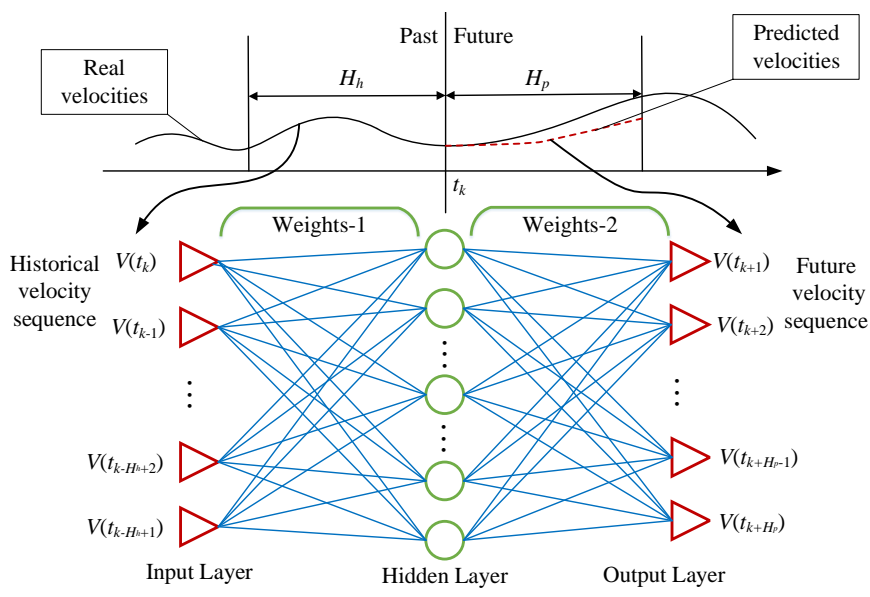

Fig. 10. Input/output pattern of the RBF-NN based horizon velocity predictor.

devices can utilize lead vehicle measurements to improve velocity prediction [41], but this is not considered in this paper.

In recent work on velocity prediction [42], artificial neural networks (ANN) have proven effective in terms of both accuracy and ease-of-use. Here, a radial basis function neural network (RBF-NN) [43] based velocity predictor is developed for short-term velocity prediction. The predicted future velocity sequence is sent to the MPC controller as the vehicle speed request in each receding horizon. Through learning from representative driving cycles, the predictor captures the highly nonlinear driving patterns in a comprehensive sense. Other velocity prediction methods, such as Gaussian mixture modeling or stochastic approaches, could also be implemented in this part [44], [42].

The input of the ANN-based predictor is a historical velocity sequence, and the output is short-term future velocity sequence $V_{\text {predict }}$, as illustrated in Fig. 10. The length of the historical velocity sequence $H_{h}$ and the length of the prediction horizon $H_{p}$ are all set as 10. Different tuning of the neural network may produce different performance. However, the trials are omitted here as this is not the focus of this paper. Interested readers may refer to [41] for more details.

Four standard driving cycles are used for the network training, including both highway and urban types: UDDS, HWFET, NEDC, US06. A real driving cycle is selected for validation from the highway driving data collected from Mobile Century. The predicted velocity sequences and the resultant root-meansquare errors (RMSE) are shown in Fig. 11. From the upper part of Fig. 11, we can see that the RBF-NN based velocity predictor predicts the micro-trip behaviors effectively. The lower part of Fig. 11 demonstrates the empirical cumulative distribution function (CDF) of the RMSE for all the prediction processes. Nearly $90 \%$ of the RMSEs are below $1.5 \mathrm{~m} / \mathrm{s}$. Considering that the prediction horizon is 10 , which is relatively long, we conclude that the RBF-NN velocity predictor is accurate enough for MPC-based PHEV energy management.
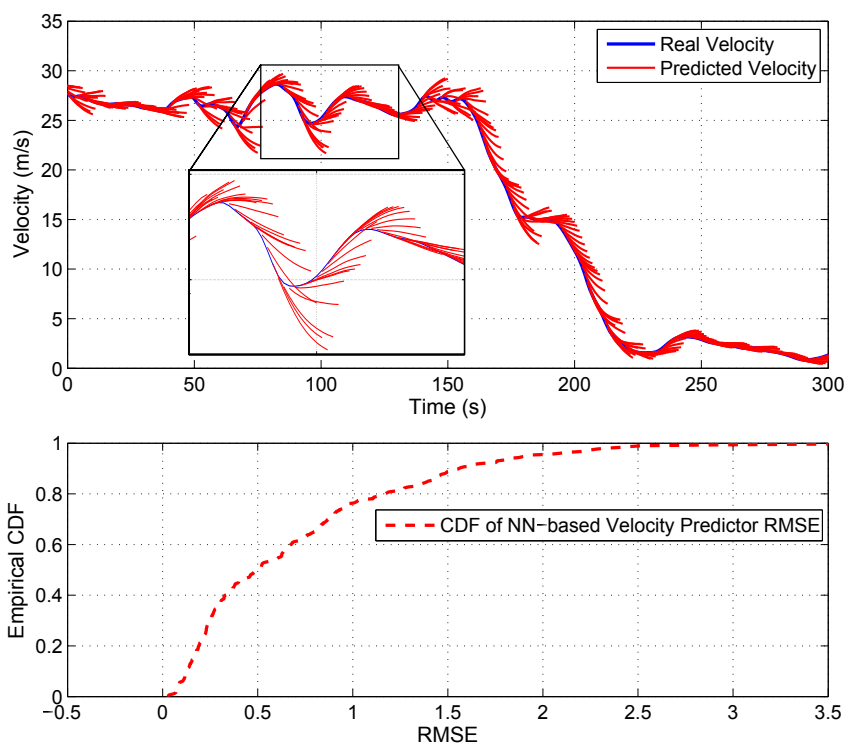

Fig. 11. Predicted velocity sequences by the RBF-NN based short-term velocity predictor, and the corresponding CDF of RMSE.

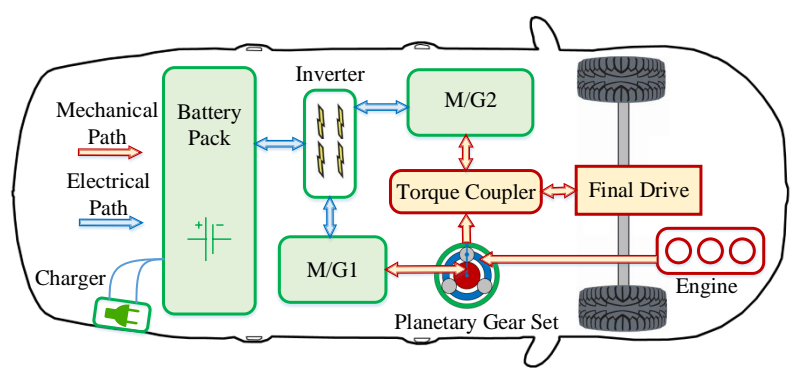

Fig. 12. Structure of the power-split PHEV configuration.

\section{MPC LEVEL CONTROL FORMULATION}

\section{A. Control-oriented PHEV Model}

A control-oriented power-split PHEV powertrain model is used in the MPC level. A planetary gear set couples the engine, $\mathrm{M} / \mathrm{G} 1$, and $\mathrm{M} / \mathrm{G} 2$. As shown in Fig. 12, the engine and $\mathrm{M} / \mathrm{G} 1$ are connected to the planet carrier and the sun gear, respectively. A torque coupler is used to connect the ring gear with $\mathrm{M} / \mathrm{G} 2$ to propel the final drive.

The schematic structure of the planetary gear set is shown in the left-hand side of Fig. 13. The right-hand side of Fig. 13 shows a lever diagram, which is commonly used to describe the kinematic constraint on the ring, carrier, and sun gear angular velocities. This constraint is given mathematically by

$$
\omega_{s} S+\omega_{r} R=\omega_{c}(S+R)
$$

where $S$ and $R$ are the radii of the sun gear and the ring gear, respectively. Angular speeds of the ring gear / M/G2, sun gear / M/G1, and carrier gear / engine are denoted as $\omega_{M / G 2}, \omega_{M / G 1}$ and $\omega_{e n g}$, respectively. They are represented by the three vectors in the lever diagram. The length and direction of the vectors denote the magnitude and direction of the respective rotational speeds. The node 'Carrier' divides 

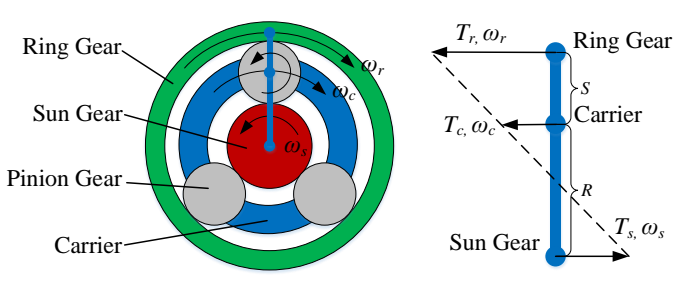

Fig. 13. Planetary gear set (left) and the lever diagram (right).

the lever into two segments, defined by the relative ratio of $S$ and $R$. The dashed line connecting the three vector arrowheads signifies that the kinematic constraint in (15) must be verified.

By neglecting the inertia of the pinion gears and assuming that all the powertrain shafts are rigid, the inertial dynamics of the powertrain are derived as

$$
\begin{aligned}
J_{M / G 1} \frac{d \omega_{M / G 1}}{d t} & =T_{M / G 1}+F S \\
J_{e n g} \frac{d \omega_{e n g}}{d t} & =T_{\text {eng }}-F(S+R) \\
J_{M / G 2} \frac{d \omega_{M / G 2}}{d t} & =T_{M / G 2}-\left(T_{\text {axle }} / g_{f}\right)+F R
\end{aligned}
$$

where $J_{M / G 1}, J_{e n g}$ and $J_{M / G 2}$ are the lumped inertias of $\mathrm{M} / \mathrm{G} 1$, engine and $\mathrm{M} / \mathrm{G} 2$, respectively; $T_{M / G 1}=T_{s}, T_{\text {eng }}=$ $T_{c}$ and $T_{M / G 2}=T_{r}$ are torques of $\mathrm{M} / \mathrm{G} 1$, engine and $\mathrm{M} / \mathrm{G} 2$, respectively; $F$ represents the internal force on pinion gears; $g_{f}$ is the gear ratio of the final drive; $T_{a x l e}$ is the torque produced from powertrain on the drive axle. To reduce the control-oriented model's complexity, we disregard the inertial dynamics, and use the steady-state values of (16)-(18). The rotational speed of $\mathrm{M} / \mathrm{G} 2$ and the axle torque are given by

$$
\begin{aligned}
\omega_{M / G 2}= & \frac{g_{f}}{R_{\text {wheel }}} V \\
m \frac{d V}{d t}= & \frac{T_{\text {axle }}+T_{\text {brake }}}{R_{\text {wheel }}}+m g \sin (\theta) \\
& -\frac{1}{2} \rho A C_{d} V^{2}-C_{r} m g \cos (\theta)
\end{aligned}
$$

where $R_{\text {wheel }}$ is the wheel radius, $T_{\text {brake }}$ is the friction brake torque and $\theta$ denotes the road grade.

At each time instant, the MPC controller computes an optimal split between the engine, M/G1, and M/G2 to minimize fuel consumption. Fuel flow rate of the engine $\left(\dot{m}_{f u e l}\right)$ and power transfer efficiencies for M/G1 and M/G2 $\left(\eta_{M / G 1}\right.$ and $\left.\eta_{M / G 2}\right)$ are extracted from empirical maps. They are all functions of angular speed and torque

$$
\begin{aligned}
\dot{m}_{\text {fuel }} & =\psi_{1}\left(\omega_{\text {eng }}, T_{\text {eng }}\right) \\
\eta_{M / G 1} & =\psi_{2}\left(\omega_{M / G 1}, T_{M / G 1}\right) \\
\eta_{M / G 2} & =\psi_{3}\left(\omega_{M / G 2}, T_{M / G 2}\right)
\end{aligned}
$$

where $\psi_{1}, \psi_{2}$ and $\psi_{3}$ are empirical maps of the engine fuel flow and $\mathrm{M} / \mathrm{G}$ efficiencies, respectively [45].

The equivalent circuit battery model is employed in the control-oriented model, and has been presented in (5) and (6). The battery in a power-split PHEV is connected to a bi-directional converter to supply power or recuperate energy from the electrical machines. Terminal battery power is described by

$P_{\text {batt }}=P_{M / G 1} /\left(\eta_{M / G 1} \eta_{i n v}\right)^{k_{M / G 1}}+P_{M / G 2} /\left(\eta_{M / G 2} \eta_{i n v}\right)^{k_{M / G 2}}$

where $P_{M / G 1}$ and $P_{M / G 2}$ are shaft powers of $\mathrm{M} / \mathrm{G} 1$ and $\mathrm{M} / \mathrm{G} 2$, respectively; $\eta_{i n v}$ is the inverter efficiency;

$$
k_{i}=\left\{\begin{array}{ll}
1 & \text { if } P_{i}>0 \\
-1 & \text { if } P_{i} \leq 0
\end{array}, \text { for } i=\{M / G 1, M / G 2\} .\right.
$$

A complete description of the battery SOC dynamics can therefore be obtained. Equations (5)-(6) and (15)-(25) summarize the control-oriented model used for MPC, and more details can be found in [25]. Throughout this study, MPC is applied to a detailed quasi-static PHEV plant [38].

\section{B. MPC Level Nonlinear Control}

The EMP in the MPC level is formulated as a constrained nonlinear optimization problem and solved by DP at each time instant [46]. Given the powertrain dynamics (15)-(25), we require two independent control inputs to render a causal system. In this paper we choose $\omega_{e n g}$ and $T_{e n g}$. Denoting $x$ as the state variable, $u$ as the control variable, $d$ as the system disturbance, and $y$ as the output, the proposed control-oriented powertrain model can be represented as

$$
\begin{aligned}
\dot{x} & =f(x, u, d) \\
y & =g(x, u, d)
\end{aligned}
$$

with $x=[\mathrm{SOC}, \mathrm{O}]^{T}, u=\left[\omega_{\text {eng }}, T_{\text {eng }}\right]^{T}, d=V_{\text {predict }}$, $y=\left[\dot{m}_{\text {fuel }}, P_{\text {batt }}, T_{M / G 2}, \omega_{M / G 1}, T_{M / G 1}\right]^{T}$. Consider a one second time step, $\Delta t=1$ second. At time step $k$, the cost function $J_{k}$ is formulated as

$$
J_{k}=\int_{k \Delta t}^{\left(k+H_{p}\right) \Delta t}\left[\dot{m}_{f u e l}(u(t))+w \delta \mathrm{O}(t)\right]^{2} d t
$$

where $H_{p}$ is the prediction horizon length, which is herein equal to the control horizon length for simplicity [20]. The disturbance $d=V_{\text {predict }}$ is predicted by the short-term velocity predictor in Section III Part C.

Additionally, the following physical constraints must be enforced:

$$
\begin{aligned}
& \mathrm{SOC}^{\min } \leq \mathrm{SOC} \leq \mathrm{SOC}^{\max }, \\
& I_{b a t t}^{\min } \leq I_{\text {batt }} \leq I_{\text {batt }}^{\max }, \quad P_{\text {batt }}^{\min } \leq P_{\text {batt }} \leq P_{b a t t}^{\max }, \\
& T_{e n g}^{\min } \leq T_{e n g} \leq T_{e n g}^{\max }, \omega_{e n g}^{\min } \leq \omega_{e n g} \leq \omega_{e n g}^{\max }, \\
& T_{M / G 1}^{\min } \leq T_{M / G 1} \leq T_{M / G 1}^{\max }, \omega_{M / G 1}^{\min } \leq \omega_{M / G 1} \leq \omega_{M / G 1}^{\max }, \\
& T_{M / G 2}^{\min } \leq T_{M / G 2} \leq T_{M / G 2}^{\max }, \omega_{M / G 2}^{\min } \leq \omega_{M / G 2} \leq \omega_{M / G 2}^{\max } .
\end{aligned}
$$

Special consideration is given to the terminal battery SOC constraint in the MPC horizon. Namely, the SOC trajectory generated from the traffic flow data in the upper level provides the terminal SOC constraint. This approach guides the SOC trajectory based on future traffic conditions without being restrictive in preventing deviations due to unknown disturbances. It is important to note that the SOC reference can be indexed in two ways: time domain dependent and space domain dependent. For time domain dependent SOC reference,

$$
\overline{\operatorname{SOC}}\left(\left(k+H_{p}\right) \Delta t\right)=\operatorname{SOC}^{*}\left(\left(k+H_{p}\right) \Delta t\right)
$$


TABLE I

General PARAmeters of THE PHEV MOdEL

\begin{tabular}{ccc}
\hline \hline Vehicle & Configuration & $\begin{array}{c}\text { Power-split } \\
\text { Curb Weight }\end{array}$ \\
& Type & Gasoline Inline 4-Cylinder \\
Engine & Max Power & $57 \mathrm{~kW} @ 4500 \mathrm{RPM}$ \\
& Max Torque & $110 \mathrm{Nm} @ 4500 \mathrm{RPM}$ \\
\hline \multirow{2}{*}{ Motor } & Type & Permanent Magnet AC \\
Generator & M/G1 Max Power & $30 \mathrm{~kW} \mathrm{@} \mathrm{3000-5500} \mathrm{RPM}$ \\
& M/G2 Max Power & $35 \mathrm{~kW} \mathrm{@} \mathrm{1040-5600} \mathrm{RPM}$ \\
\hline \multirow{3}{*}{ Battery Pack } & Cell Chemistry & $\mathrm{C}-\mathrm{LiFePO} 4$ \\
& Energy Capacity & $5 \mathrm{kWh}$ for pack \\
& Charge Capacity & $2.3 \mathrm{Ah}$ per Cell \\
& Cell Number & 660 \\
& Cell Arrangement & $110 \mathrm{~S} \times 6 \mathrm{P}$ \\
\hline \hline
\end{tabular}

For space domain dependent SOC reference,

$$
\overline{\operatorname{SOC}}\left(n_{k} \Delta s\right)=\operatorname{SOC}^{*}\left(n_{k} \Delta s\right)
$$

where $\Delta s$ is the space step, $n_{k}$ indicates the space step number that the vehicle drives through by the end of time horizon $k$. $\mathrm{SOC}^{*}$ is the optimal SOC trajectory generated from the supervisory SOC planning level in Section III Part B. Both indexing methods are investigated in the following section.

\section{Simulation Results And Discussion}

The proposed power balance model and predictive energy management framework are both evaluated in this section. All the simulations were performed on a personal computer with an Intel Corel i7-3630QM CPU @ 2.4GHz. The power-split PHEV structure is adopted from [47], and general parameters of the model are provided in Table I. The empirical fuel consumption map of the engine and the efficiency maps of the motor/generator are from ADVISOR [45]. Note that in the simulation and validation subsections, we focus on trips that exceed the all-electric-range, and therefore require engine power at some point.

\section{A. Power Balance Model Validation}

The power balance model is validated by comparing the generated SOC trajectory with the control-oriented model. Based on the approximated $\eta_{1}$ and $\eta_{2}$ from Fig. 8 and Fig. 9, the optimization problem (8) can be solved by DP. The initial battery SOC is set as 0.7 and terminal SOC is set as 0.3 . Six standard driving cycles and two real-world cycles, highwayReal $_{h}$ and urban-Real ${ }_{u}$ (see Fig. 14 bottom), are combined arbitrarily to construct three longer trips for testing. Notice that the validation driving cycles are different from those used to identify $\eta_{1}$ and $\eta_{2}$. Details of the trips are shown in the upper part of Table II.

The computation time of DP highly depends on the grid resolution of the control or state variables during discretization. For the control-oriented model, the authors carefully tuned the grid numbers of the control variables $\left(\omega_{\text {eng }}\right.$ and $T_{\text {eng }}$ ) and the state variable (SOC) as 40,30 and 30 , respectively.
TABLE II

TRIP DETAILS \& SOC TRAJECTORY COMPARISON

\begin{tabular}{c|c|c}
\hline \hline Trip No./Type & Composed by Cycles & Length $(\mathrm{s} / \mathrm{km})$ \\
\hline 1/Urban & UDDS NEDC Real & \\
2/Highway & HWFET WVUINTER Real & $3528 / 32.8$ \\
3/Mixed & WVUSUB USO6 Real $_{h}$ Real $_{u}$ & $4112 / 65.0$ \\
& \multicolumn{3}{|c}{$4820 / 56.3$} \\
\hline
\end{tabular}

\begin{tabular}{ccccc}
\hline \hline Trip No./Model & Avg $|e|$ & Max $|e|$ & $T(\mathrm{~s})$ & SOC \\
\hline 1/C.O. & - & - & $197.2(100 \%)$ & 0.30 \\
1/P.B. & 0.0147 & 0.0249 & $31.8(16.1 \%)$ & 0.30 \\
2/C.O. & - & - & $231.9(100 \%)$ & 0.30 \\
2/P.B. & 0.0226 & 0.0467 & $37.5(16.2 \%)$ & 0.30 \\
3/C.O. & - & - & $261.1(100 \%)$ & 0.30 \\
3/P.B. & 0.0168 & 0.0438 & $41.2(15.8 \%)$ & 0.30
\end{tabular}

'C.O.' and 'P.B.' denote the control-oriented model and the power balance model, respectively. SOC denotes the terminal SOC value.

This resolution is a compromise between the optimality and the computational complexity. For fair comparison, the control variable $\left(P_{b a t t}\right)$ and state variable (SOC) in the power balance model are also set as 40 and 30, respectively.

The bottom part of Table II demonstrates the comparison details of generating SOC trajectory from the two PHEV models. Symbol $T$ is the computation time. As can be seen, for trips that exceed the all-electric-range, the control-oriented model requires 190-260 seconds to compute the optimal SOC trajectory. Considering that the traffic flow updating rate is 300 seconds, it may be difficult for the control-oriented model to satisfy the real-time traffic data updating requirement on an embedded system. However, the computation time required by the power balance model is $80 \%$ less than the control-oriented model. This indicates that the power balance model facilitates optimal SOC trajectory generation, and is computationally sufficient for rapid SOC trajectory calculation.

Denote the SOC error as $e(t)$, thus,

$$
e(t)=\operatorname{SOC}_{p}(t)-\operatorname{SOC}_{c}(t)
$$

where $\mathrm{SOC}_{p}(t)$ and $\mathrm{SOC}_{c}(t)$ are the SOC trajectories calculated from the power balance model and the control-oriented model, respectively. It can be seen from Table II that both models guarantee the requested terminal SOC constraint. The average and maximal SOC errors between the power balance model and control-oriented model remain within 0.03 and 0.05 , which are $3 \%$ and $5 \%$ of the battery SOC full scale, respectively. This suggests that the power balance model is reasonably accurate for the optimal SOC trajectory calculation, and therefore serves the SOC planning purpose at the top level of Fig. 6. The resultant battery SOC trajectories of the 3/Mixed driving trip testing are illustrated in Fig. 14. As can be seen, the SOC trajectory of the power balance model follows the control-oriented model well.

\section{B. Energy Management Strategy Evaluation}

The congestion period from 10:20 AM to 13:40 PM in the obtained traffic flow data is chosen to evaluate the proposed energy management strategy. Due to the experimental field's limited size, most of the individual trips are no more than ten 

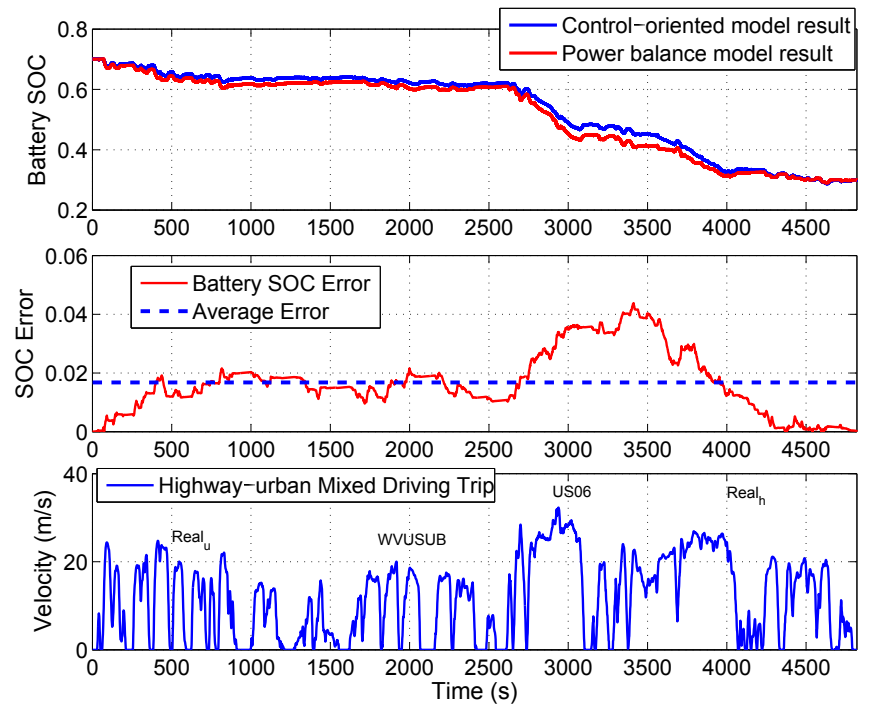

Fig. 14. Comparison of SOC trajectory in the 3/Mixed case. From top to bottom: SOC trajectories, the absolute SOC error $|e|$ and the driving profile. The average $|e|$ is 0.0168 (1.68\% of the battery SOC full scale).

miles long, which is shorter than the all-electric-range. In this case, 24 arbitrarily selected individual driving trips are concatenated into six longer cycles for testing, as shown in Fig. 15, and each testing cycle includes one or two traffic congestions. The corresponding traffic conditions are assumed to update sequentially. The SOC reference is computed simultaneously by the controller. In practice, the system must access realtime traffic information from the cloud/Internet, and the SOC generation can therefore be performed in the cloud during this process.

All of the real driving profiles are completely blind to all the MPC simulations. The same RBF-NN velocity predictor is used for short-term velocity prediction. For simplicity, the control and prediction horizons are both set as 10 steps, as a compromise between control performance and the computation complexity. The average computation time of the simulation process at each time instant (one second) is 0.6-0.7 seconds. Therefore, the proposed energy management strategy is potentially implementable on an embedded system.

Based on different traffic data accessibility levels, five situations are considered to evaluate the proposed supervised predictive energy management strategy:

1) CDCS: When no traffic information is available, the CDCS strategy is used for battery SOC planning.

2) Static@T: Static traffic information is available and the vehicle obtains the traffic velocity information only once at the beginning of the trip. The first generated SOC reference is assumed to be relevant until the end of the trip, and is indexed by time.

3) Static@S: Static traffic information is available and the SOC reference is indexed by space.

4) Dynamic@T: Dynamic traffic information is available and the vehicle obtains the traffic velocity information every 300 seconds. The generated SOC reference is indexed by time.
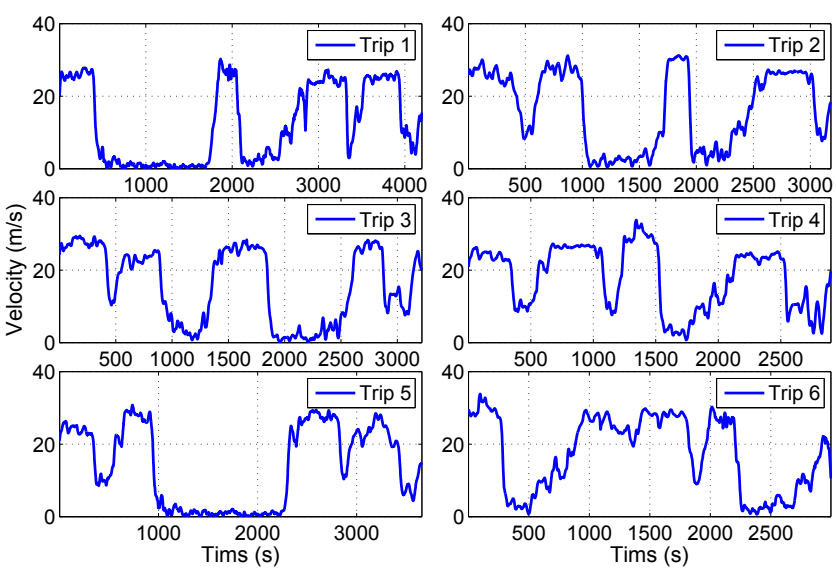

Fig. 15. Six assembled driving cycles from 24 arbitrarily selected trips from 10:20 AM to 13:40 PM of the Mobile Century experiment data.

5)Dynamic@S: Dynamic traffic information is available, and the SOC reference is indexed by space.

The resultant SOC trajectories of these five situations for testing Trip 2 (from Fig. 15) are demonstrated in Fig. 16, including deterministic DP as a benchmark. As can be seen, the CDCS strategy consumes the battery energy within 900 sec, and then sustains SOC around 0.3 for the remainder of the trip. In the Static@T scenario, the battery energy is not completely consumed by the end of the trip. Thus, the terminal battery SOC constraint is not satisfied. If the generated SOC reference is indexed by space, such as in the Static@S case, the terminal SOC constraint is satisfied. This is because the driving cycle extracted from the traffic data has different time length, but the same spatial length with the real driving cycle. On the other hand, if the traffic status updates continuously, the supervised predictive energy management can produce nearly optimal performance. The SOC trajectory errors caused from outdated traffic data can be corrected by updated data. The terminal SOC requirement is satisfied in both the Dynamic@T and Dynamic@S cases. The resultant battery SOC trajectory for the Dynamic@S approach is nearly optimal with respect to the DP benchmark, which can be clearly seen in Fig. 16 .

The deviation between the final battery SOC and the desired value has been compensated in the fuel consumption calculations, denoted as $\mathrm{Fuel}_{c}$. Detailed simulation results for Trip 2 are shown in Table III. As expected, the fuel consumption of predictive energy management with dynamic traffic information available is less than the CDCS strategy and the static traffic information approaches. Namely, they achieve 95.1\% (Dynamic@T) and 95.9\% (Dynamic@S) fuel economy optimality of the DP benchmark. It is also interesting to note that a space-domain-indexed SOC reference consistently consumes less fuel than the time-domain-indexed SOC reference, in both the static and dynamic traffic information situations.

Simulation results for all six testing trips from Fig. 15 are illustrated in Fig. 17, including the average value and standard deviation of the fuel optimality and the terminal SOC. DP, with full knowledge of the trip, achieves $100 \%$ fuel optimality and absolute 0.3 terminal SOC for all tests. The CDCS strategy 
TABLE III

SIMULATION RESULTS FOR TRIP 2

\begin{tabular}{cccc}
\hline \hline Type & Terminal SOC & Fuel $_{c}(\mathrm{~g})$ & Fuel Optimality \\
\hline DDP & 0.3000 & 628.5 & $100 \%$ \\
CDCS & 0.2955 & 691.6 & $89.9 \%$ \\
Static@T & 0.3911 & 712.2 & $86.7 \%$ \\
Static@S & 0.2965 & 678.9 & $92.0 \%$ \\
Dynamic@T & 0.2895 & 659.1 & $95.1 \%$ \\
Dynamic@S & 0.2971 & 654.5 & $95.9 \%$ \\
\hline
\end{tabular}
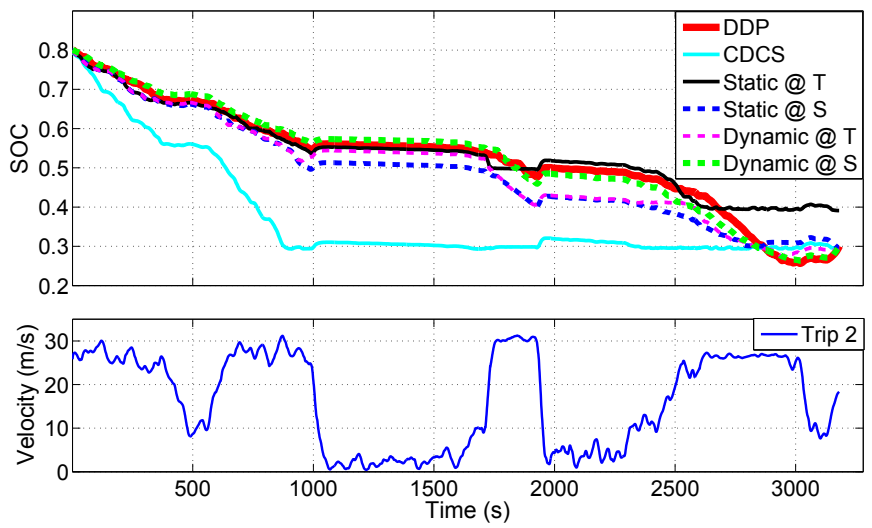

Fig. 16. Battery SOC trajectories of Trip 2. The Dynamic@T and Dynamic@S cases produce nearly optimal SOC trajectories.

maintains an average of $90 \%$ fuel optimality, and the terminal SOC is always restrained around 0.3. Due to the poor accuracy of the initial traffic flow velocity in estimating the actual driving cycle, the Static@T approach performs worse than the CDCS approach on average. In particular, the terminal SOC has a relatively high variance around 0.3 . By indexing the SOC reference in space, Static@S guarantees the terminal SOC constraint is respected with low variance. As a result, the overall fuel economy is improved by $5 \%$ on average.

In the Dynamic@T situation, the deviation of the terminal SOC is reduced by updating the traffic data every 300 seconds compared with the Static@T approach. The terminal SOC is restricted within an acceptable range between 0.3 and 0.35 . More importantly, nearly $94 \%$ average fuel optimality is achieved with dynamic traffic data enabled, which is a considerable improvement considering the uncertainty of the driving cycles. By indexing the SOC reference within the spatial domain, the terminal SOC constraint is better respected and the fuel consumption is further reduced in the Dynamic@S case. This phenomenon is consistent with the results derived from the static traffic situations.

The demonstrated results are conducted from a highway congestion-involved driving scenario constructed by the Mobile Century experiment database. Different results could be observed with different driving trips or in different traffic conditions used. However, the proposed predictive energy management proves to be effective in achieving near optimal fuel economy with traffic feedback data.

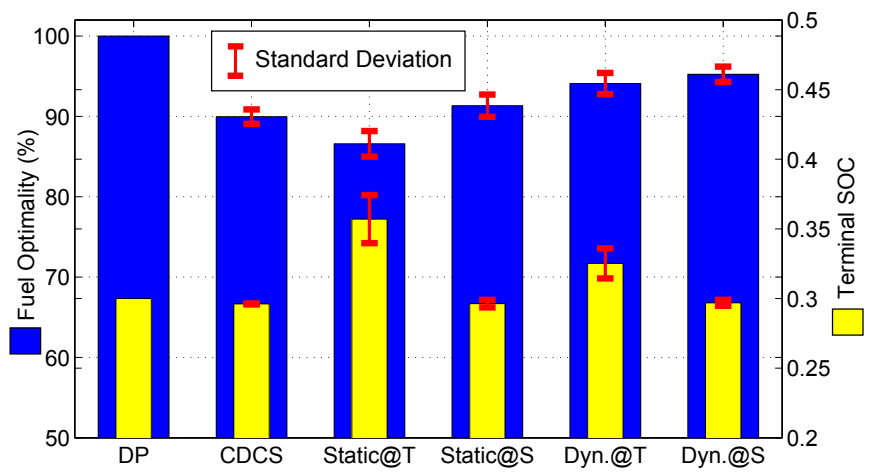

Fig. 17. Average fuel optimality and terminal SOC results for all the testing trips. Dynamic traffic information allows the proposed energy management strategy to achieve nearly $95 \%$ fuel optimality while consistently ensuring the terminal SOC value.

\section{CONCLUSIONS}

This paper presents a predictive PHEV energy management strategy that integrates real-time traffic flow velocity data. The strategy is composed of a two-tiered scheme. A supervisory SOC planning level rapidly generates an SOC trajectory from traffic data for the terminal SOC constraints in the MPC level. A power balance PHEV model is developed for this upper-level. With this model, DP computes the optimal SOC trajectory in real-time - at a rate commensurate with traffic data update rates $(300 \mathrm{sec})$. Simulation results show that the predictive energy management strategy with dynamic traffic data can achieve $94-96 \%$ fuel optimality of the deterministic DP benchmark in a highway driving scenario, despite congestion events. Future work involves validation of the proposed energy management strategy through a hardware-in-the-loop experiment, and considerations for battery health in the cost function.

\section{ACKNOWLEDGMENT}

This work was supported by the National High Technology Research and Development Program of China (2011AA11228) in part, and the National Sci-Tech Support Plan (2013BAG05B00) in part. The authors would also like to thank Prof. Alexandre M. Bayen at the University of California, Berkeley, USA, for substantial help in providing the traffic data.

\section{REFERENCES}

[1] A. R. Beresford and J. Bacon, "Intelligent transportation systems," Pervasive Computing, IEEE, vol. 5, no. 4, pp. 63-67, 2006.

[2] C. N. E. Anagnostopoulos, I. E. Anagnostopoulos, V. Loumos, and E. Kayafas, "A license plate-recognition algorithm for intelligent transportation system applications," Intelligent Transportation Systems, IEEE Transactions on, vol. 7, no. 3, pp. 377-392, 2006.

[3] R. Cucchiara, M. Piccardi, and P. Mello, "Image analysis and rule-based reasoning for a traffic monitoring system," Intelligent Transportation Systems, IEEE Transactions on, vol. 1, no. 2, pp. 119-130, 2000.

[4] C. Chen, K. Petty, A. Skabardonis, P. Varaiya, and Z. Jia, "Freeway performance measurement system: mining loop detector data," Transportation Research Record: Journal of the Transportation Research Board, vol. 1748, no. 1, pp. 96-102, 2001.

[5] V. Briggs and K. Jasper, "Organizing for regional transportation operations: Houston TranStar,” Tech. Rep., 2001. 
[6] J. Miller, S.-i. Kim, M. Ali, and T. Menard, "Determining time to traverse road sections based on mapping discrete GPS vehicle data to continuous flows," in Intelligent Vehicles Symposium (IV). IEEE, 2010, pp. 615-620.

[7] Y. Zhao, "Mobile phone location determination and its impact on intelligent transportation systems," Intelligent Transportation Systems, IEEE Transactions on, vol. 1, no. 1, pp. 55-64, 2000.

[8] C. Zhang, A. Vahidi, P. Pisu, X. Li, and K. Tennant, "Role of terrain preview in energy management of hybrid electric vehicles," Vehicular Technology, IEEE Transactions on, vol. 59, no. 3, pp. 1139-1147, 2010.

[9] Q. Gong, Y. Li, and Z.-R. Peng, "Trip-based optimal power management of plug-in hybrid electric vehicles," Vehicular Technology, IEEE Transactions on, vol. 57, no. 6, pp. 3393-3401, 2008.

[10] H. Yu, M. Kuang, and R. McGee, "Trip-oriented energy management control strategy for plug-in hybrid electric vehicles," in Decision and Control and European Control Conference (CDC-ECC), 50th IEEE Conference on. IEEE, 2011, pp. 5805-5812.

[11] M. Ehsani, Y. Gao, and A. Emadi, Modern electric, hybrid electric, and fuel cell vehicles: fundamentals, theory, and design. CRC press, 2009.

[12] P. B. Sharer, A. Rousseau, D. Karbowski, and S. Pagerit, Plug-in hybrid electric vehicle control strategy: comparison between EV and chargedepleting options. SAE International, 2008.

[13] P. Tulpule, V. Marano, and G. Rizzoni, "Effects of different PHEV control strategies on vehicle performance," in American Control Conference (ACC). IEEE, 2009, pp. 3950-3955.

[14] P. Pisu and G. Rizzoni, "A comparative study of supervisory control strategies for hybrid electric vehicles," Control Systems Technology, IEEE Transactions on, vol. 15, no. 3, pp. 506-518, 2007.

[15] S. G. Wirasingha and A. Emadi, "Classification and review of control strategies for plug-in hybrid electric vehicles," vehicular Technology, IEEE Transactions on, vol. 60, no. 1, pp. 111-122, 2011.

[16] D. Ambuhl and L. Guzzella, "Predictive reference signal generator for hybrid electric vehicles," Vehicular Technology, IEEE Transactions on, vol. 58, no. 9, pp. 4730-4740, 2009.

[17] A. Sciarretta and L. Guzzella, "Control of hybrid electric vehicles," Control systems, IEEE, vol. 27, no. 2, pp. 60-70, 2007.

[18] C.-C. Lin, H. Peng, J. W. Grizzle, and J.-M. Kang, "Power management strategy for a parallel hybrid electric truck," Control Systems Technology, IEEE Transactions on, vol. 11, no. 6, pp. 839-849, 2003.

[19] L. Serrao, S. Onori, and G. Rizzoni, "ECMS as a realization of pontryagin's minimum principle for HEV control," in American Control Conference (ACC). IEEE, 2009, pp. 3964-3969.

[20] H. Borhan, A. Vahidi, A. M. Phillips, M. L. Kuang, I. V. Kolmanovsky, and S. Di Cairano, "MPC-based energy management of a power-split hybrid electric vehicle," Control Systems Technology, IEEE Transactions on, vol. 20, no. 3, pp. 593-603, 2012.

[21] J. Liu and H. Peng, "Modeling and control of a power-split hybrid vehicle," Control Systems Technology, IEEE Transactions on, vol. 16, no. 6, pp. 1242-1251, 2008.

[22] K. Çağatay Bayindir, M. A. Gözüküçük, and A. Teke, “A comprehensive overview of hybrid electric vehicle: Powertrain configurations, powertrain control techniques and electronic control units," Energy Conversion and Management, vol. 52, no. 2, pp. 1305-1313, 2011.

[23] L. V. Pérez, G. R. Bossio, D. Moitre, and G. O. García, “Optimization of power management in an hybrid electric vehicle using dynamic programming," Mathematics and Computers in Simulation, vol. 73, no. 1 , pp. 244-254, 2006.

[24] L. Johannesson, M. Asbogard, and B. Egardt, "Assessing the potential of predictive control for hybrid vehicle powertrains using stochastic dynamic programming," Intelligent Transportation Systems, IEEE Transactions on, vol. 8, no. 1, pp. 71-83, 2007.

[25] S. J. Moura, H. K. Fathy, D. S. Callaway, and J. L. Stein, “A stochastic optimal control approach for power management in plug-in hybrid electric vehicles," Control Systems Technology, IEEE Transactions on, vol. 19, no. 3, pp. 545-555, 2011.

[26] F. A. Bender, M. Kaszynski, and O. Sawodny, "Drive cycle prediction and energy management optimization for hybrid hydraulic vehicles," Vehicular Technology, IEEE Transactions on, vol. 62, no. 8, pp. 35813592, 2013

[27] C. Musardo, G. Rizzoni, Y. Guezennec, and B. Staccia, "A-ECMS:
[27] C. Musardo, G. Rizzoni, Y. Guezennec, and B. Staccia, "A-ECMS: An adaptive algorithm for hybrid electric vehicle energy management," European Journal of Control, vol. 11, no. 4, pp. 509-524, 2005.

[28] N. Kim, S. Cha, and H. Peng, "Optimal control of hybrid electric vehicles based on pontryagin's minimum principle," Control Systems Technology, IEEE Transactions on, vol. 19, no. 5, pp. 1279-1287, 2011

[29] B. Gu and G. Rizzoni, "An adaptive algorithm for hybrid electric vehicle energy management based on driving pattern recognition," in ASME International Mechanical Engineering Congress and Exposition. ASME, 2006, pp. 249-258.

[30] E. F. Camacho, C. Bordons, E. F. Camacho, and C. Bordons, Model predictive control. Springer London, 2004, vol. 2.

[31] P. Falcone, F. Borrelli, J. Asgari, H. E. Tseng, and D. Hrovat, "Predictive active steering control for autonomous vehicle systems," Control Systems Technology, IEEE Transactions on, vol. 15, no. 3, pp. 566-580, 2007.

[32] B. Asadi and A. Vahidi, "Predictive cruise control: Utilizing upcoming traffic signal information for improving fuel economy and reducing trip time," Control Systems Technology, IEEE Transactions on, vol. 19, no. 3 , pp. 707-714, 2011.

[33] K. Uthaichana, S. Bengea, R. DeCarlo, S. Pekarek, and M. Zefran, "Hybrid model predictive control tracking of a sawtooth driving profile for an HEV," in American Control Conference (ACC). IEEE, 2008, pp. 967-974.

[34] G. Ripaccioli, A. Bemporad, F. Assadian, C. Dextreit, S. Di Cairano, and I. V. Kolmanovsky, "Hybrid modeling, identification, and predictive control: An application to hybrid electric vehicle energy management," in Hybrid Systems: Computation and Control. Springer, 2009, pp. 321-335.

[35] V. Ngo, T. Hofman, M. Steinbuch, and A. Serrarens, "Predictive gear shift control for a parallel hybrid electric vehicle," in Vehicle Power and Propulsion Conference (VPPC). IEEE, 2011, pp. 1-6.

[36] L. Johannesson and B. Egardt, "A novel algorithm for predictive control of parallel hybrid powertrains based on dynamic programming," in Advances in Automotive Control, vol. 5, no. 1, 2007, pp. 343-350.

[37] J. C. Herrera, D. B. Work, R. Herring, X. J. Ban, Q. Jacobson, and A. M. Bayen, "Evaluation of traffic data obtained via GPS-enabled mobile phones: The mobile century field experiment," Transportation Research Part C: Emerging Technologies, vol. 18, no. 4, pp. 568-583, 2010.

[38] L. Guzzella and A. Amstutz, "CAE tools for quasi-static modeling and optimization of hybrid powertrains," Vehicular Technology, IEEE Transactions on, vol. 48, no. 6, pp. 1762-1769, 1999.

[39] X. Hu, S. Li, and H. Peng, "A comparative study of equivalent circuit models for Li-ion batteries," Journal of Power Sources, vol. 198, pp. 359-367, 2012.

[40] M. André, "The ARTEMIS european driving cycles for measuring car pollutant emissions," Science of the total Environment, vol. 334, pp. 73-84, 2004.

[41] C. Sun, X. Hu, S. Moura, and F. Sun, "Comparison of velocity forecasting strategies for predictive control in HEVs," in Dynamic Systems and Control Conference (DSCC). ASME, 2014, accepted, paper number 6031.

[42] S. Lefvre, C. Sun, R. Bajcsy, and C. Laugier, "Comparison of parametric and non-parametric approaches for vehicle speed prediction," in American Control Conference (ACC). IEEE, 2014, pp. 3494-3499.

[43] M. T. Hagan, H. B. Demuth, M. H. Beale et al., Neural network design. Pws Pub. Boston, 1996.

[44] G. Ripaccioli, D. Bernardini, S. Di Cairano, A. Bemporad, and I. Kolmanovsky, "A stochastic model predictive control approach for series hybrid electric vehicle power management," in American Control Conference $(A C C)$. IEEE, 2010, pp. 5844-5849.

[45] T. Markel, A. Brooker, T. Hendricks, V. Johnson, K. Kelly, B. Kramer, M. OKeefe, S. Sprik, and K. Wipke, "ADVISOR: a systems analysis tool for advanced vehicle modeling," Journal of power sources, vol. 110, no. 2, pp. 255-266, 2002.

[46] O. Sundstrom and L. Guzzella, "A generic dynamic programming matlab function," in Control Applications,(CCA) \& Intelligent Control,(ISIC). IEEE, 2009, pp. 1625-1630.

[47] K. Muta, M. Yamazaki, and J. Tokieda, "Development of new-generation hybrid system THS II-Drastic improvement of power performance and fuel economy," SAE paper, no. 01, pp. 64-75, 2004. 